\title{
Effects of Cirsium setidens (Dunn) Nakai on the osteogenic differentiation of stem cells
}

\author{
SAYAN DEB DUTTA ${ }^{1 *}$, DINESH K. PATEL ${ }^{1 *}$, BIN JIN $^{2 *}$, SUN-IL CHOI $^{3}$, OK HWAN LEE $^{3}$ and KI-TAEK LIM ${ }^{1}$ \\ ${ }^{1}$ Department of Biosystems Engineering, Kangwon National University, Chuncheon, Gangwon 24341, Republic of Korea; \\ ${ }^{2}$ Department of Stomatology, Affiliated Hospital of Yanbian University, Yanji, Jilin 133000, P.R. China; ${ }^{3}$ Department of \\ Food Science and Biotechnology, Kangwon National University, Chuncheon, Gangwon 24341, Republic of Korea
}

Received August 1,2020; Accepted November 2, 2020

DOI: $10.3892 / \mathrm{mmr} .2021 .11903$

\begin{abstract}
Cirsium setidens (Dunn) Nakai, commonly known as gondre, is a perennial herb that grows predominantly in South Korea. It contains several bioactive phytochemicals with antioxidant, anti-cancer, anti-tumor and anti-inflammatory properties. The present study aimed to investigate the effects of methanolic extracts of gondre on osteogenic differentiation of human periodontal ligament stem cells (hPDLSCs). As characterized by nuclear magnetic resonance spectroscopy and matrix-assisted laser deposition/ionization (time-of-flight) mass spectrometry, the methanol extract of gondre was found to be enriched with pectolinarin. After $48 \mathrm{~h}$, enhanced viability of hPDLSCs was observed in the presence of gondre compared with under control conditions, suggesting the biocompatibility of gondre. Notably, biocompatibility was markedly affected by gondre concentration in cultured media. Relatively high cell viability was observed in medium containing $0.05 \%$ gondre. Furthermore, mineralization was significantly higher in hPDLSCs in the presence of gondre compared with that in control cells, indicating their mineralization potential. Increased expression of various transcription markers, such as collagen 1, runt-related transcription factor 2, bone sialoprotein and alkaline phosphatase, was also detected when hPDLSCs were stimulated with gondre compared with in the control groups, further confirming the superior osteogenic potential of gondre extract for tissue engineering applications, particularly in bone tissues.
\end{abstract}

Correspondence to: Professor Ki-Taek Lim, Department of Biosystems Engineering, Kangwon National University, 1-Kangwondaehak-gil, Chuncheon, Gangwon 24341, Republic of Korea

E-mail: ktlim@kangwon.ac.kr

${ }^{*}$ Contributed equally

Key words: Cirsium setidens, pectolinarin, cell viability, osteogenesis, tissue engineering

\section{Introduction}

Medicinal plants and their derivatives have emerged as functional food materials globally because they contain diverse bioactive phytochemicals with potential immunomodulatory effects on the human body. Therefore, the demand for functional foods and beverages has rapidly increased $(1,2)$. Active ingredients in functional foods include polyunsaturated fatty acids, peptides, flavonoids, and phenolic compounds, which help prevent obesity, diabetes, and cancer $(3,4)$.

Cirsium setidens (Dunn) Nakai, a wild perennial herb popularly known as gondre, is mainly found in the Kangwon province of South Korea (5). Its extract is commonly used as a food product in Korea, China, Japan, Canada, USA, and Australia (6). All parts of Cirsium spp., including roots, are enriched in phytochemicals, such as flavonoids, phenolics, sterols, and alkaloids, and utilized in the treatment of liver and kidney inflammation and bleeding, as well as other hepatic disorders (7-9). In addition, the aerial parts of $C$. chanroenicum are used to treat fever and to improve detoxification and blood circulation, in Chinese medicine (10). Leaves and stems are rich sources of calcium, proteins, and vitamins. In the case of gondre, pectolinarin, pectolinarigenin, apigenin, and luteolin are the essential phytochemicals that can regulate various physiological activities (11). Pectolinarin has anti-tumor, anti-cancer, anti-oxidant, anti-inflammatory, and hepatoprotective properties, whereas pectolinarigenin has anti-oxidant and anti-inflammatory effects (12-14).

Stem cells are considered a promising source for regenerative medicine and tissue engineering, because of their multi-lineage differentiation potential and self-renewal properties $(15,16)$. For instance, human mesenchymal stem cells (hMSCs) are used in cell-based therapies to treat various diseases. Nevertheless, microenvironment conditions, such as oxidative stress, low oxygen level, inflammation, and limited nutrient supply, restrict clinical trials of stem cells (17).

Human dental mesenchymal stem cells can be obtained from different dental tissues, such as the periodontal ligament, dental pulp, periapical follicle, and apical papilla. These cells have the potential for multi-lineage differentiation, and can be exploited to regenerate a desired cell type for tissue engineering applications $(18,19)$. 
This study aimed to investigate the differentiation potential of human periodontal ligament stem cells (hPDLSCs) in the presence of gondre powder. Thus, a methanol extraction was performed to determine the major bioactive components of the gondre powder, and the extracted material was analyzed with nuclear magnetic resonance (NMR) and matrix-assisted laser deposition/ionization (time-of-flight) MALDI-TOF mass spectrometry. The results indicated that pectolinarin is the chief component of the gondre extract. Enhanced mineralization occurred in hPDLSCs in the presence of gondre powder via the activation of osteogenesis-related genes and proteins. Therefore, gondre can be used as a functional food material for improving metabolism and cellular homeostasis.

\section{Materials and methods}

Extraction and characterization of the methanol extract of $C$. setidens. The gondre powder was obtained from the Department of Food Science and Biotechnology, Kangwon National University, Republic of Korea. The extraction of bioactive phytochemicals was performed as described by Jeong et al (11). Briefly, methanol was added to sufficient quantities of dried gondre powder at room temperature, with continuous mechanical stirring for $4 \mathrm{~h}$. After that, the mixture was filtered to remove undesired particles, and repeated extraction was performed three times. The obtained solution was centrifuged $\left(4,000 \mathrm{xg} / 10^{\circ} \mathrm{C}\right)$ for $30 \mathrm{~min}$, and the supernatant was separated for further analysis. The chemical structures of the phytochemicals present in the methanol extract were elucidated with ${ }^{1} \mathrm{H}-\mathrm{NMR}$ (JNM-ECZ400S/L1) in DMSO- $\mathrm{d}_{6}$ solvent, and MALDI-TOF mass spectrometry (Bruker Autoflex speed TOF/TOF).

Culture of hPDLSCs. Human third molars were collected from three young males (18-22 years). This protocol was approved by the Institutional Review Board of the Dental Hospital, Seoul National University (Seoul, Republic of Korea; IRB Number 05004), and written consent was obtained from each patient. The primary cell culture was established according to the procedure described by Jin and Choung (20). In brief, hPDLSCs were gradually separated from the extracted third molar and treated with a solution of $3 \mathrm{mg} / \mathrm{ml}$ collagenase type 1 (Worthington Biochem) and $4 \mathrm{mg} / \mathrm{ml}$ dispase (Boehringer-Mannheim) at $37^{\circ} \mathrm{C}$ for $1 \mathrm{~h}$. The cell suspension was obtained by passing the solution through a $40-\mu \mathrm{m}$ strainer (Falcon-BD Labware). The derived cells were cultured in alpha-modified Eagle's medium ( $\alpha$-MEM, Gibco; Thermo Fisher Scientific, Inc.) supplemented with $10 \%$ fetal bovine serum (FBS; Gibco; Thermo Fisher Scientific, Inc.), $100 \mu \mathrm{mol} / 1$ ascorbic acid 2-phosphate (Sigma-Aldrich; Merck $\mathrm{KGaA}$ ), $2 \mathrm{mM}$ glutamine, $100 \mathrm{U} / \mathrm{ml}$ penicillin, and $100 \mu \mathrm{g} / \mathrm{ml}$ streptomycin (Biofluids), and incubated at $37^{\circ} \mathrm{C}$ in $5 \% \mathrm{CO}_{2}$. The medium was changed after $24 \mathrm{~h}$, followed by changes every 3-4 days. Only primary cells at passages 2 or 3 were used for the proliferation and differentiation studies.

Flow cytometry analysis. The expression of mesenchymal stem cell-associated surface markers at passage 3 was analyzed with flow cytometry, to characterize the immunophenotype. Approximately $1 \times 10^{6}$ cells were fixed with $3.7 \%$ paraformal- dehyde (Sigma-Aldrich; Merck KGaA) for 10 min and then re-suspended in phosphate-buffered saline (PBS) solution (Welegene) containing $1 \%$ bovine serum albumin (BSA) (ICN Biomedicals), for $30 \mathrm{~min}$ to block the nonspecific antibody-binding sites. Next, the cells were incubated with specific antibodies against CD34, CD13, CD90, and CD146 at $4^{\circ} \mathrm{C}$ for $1 \mathrm{~h}$, followed by incubation with fluorescent secondary antibodies at room temperature for $1 \mathrm{~h}$. All antibodies were purchased from BD Biosciences. The percentages of CD13, CD90, and CD146-positive, as well as CD34-negative cells, were measured with a fluorescence-activated cell sorting (FACS) caliber flow cytometer (Becton Dickinson Immunocytometry Systems). The data were analyzed using the Cell Quest Pro software (BD Biosciences ).

Cytotoxicity and migration assay. Cell proliferation and cytotoxicity were measured using the colorimetric 3-(4,5-dimethylthazol-2-yl)-2,5-diphenyltetrazolium bromide (MTT) assay kit (Promega Corp.). Briefly, the cells were seeded in 96 -well plates $\left(1 \times 10^{4}\right.$ cells per well), cultured for the desired periods, and then treated with various concentrations of gondre powder $(0.01,0.05,0.1,0.2$, and $0.25 \%)$ for the required time periods. These concentrations have proliferative effects, as reported earlier (21). Medium without gondre was considered a negative control. The dye solution $(15 \mu \mathrm{l})$ was added at the end of the treatment, followed by further incubation in $5 \% \mathrm{CO}_{2}$ at $37^{\circ} \mathrm{C}$ for $4 \mathrm{~h}$. The solubilized formazan product was obtained by adding $100 \mu \mathrm{l}$ of a solubilization/stop solution in the media. The formed products were quantitated using an ELISA plate reader at $595 \mathrm{~nm}$ (with readings at $655 \mathrm{~nm}$ as reference). All experiments were performed in triplicate, and values are expressed as mean \pm standard deviation (SD).

We performed migration assays to assess the defect healing potential of hPDLSCs in the presence of gondre. To this end, cells were seeded in 6-well plates and allowed to grow to 90-100\% confluence. Next, the cell monolayer was wounded with a plastic tip $(1 \mathrm{~mm})$, and washed with PBS (twice) to remove cell debris. Scratched cells were incubated either with or without gondre. Cell migration towards the wounded area was monitored after time intervals of $0,12,24$ and $48 \mathrm{~h}$, using a light microscope (Olympus U-SPT; Olympus Corporation). Cell migration was quantified by measuring the distance traveled by migrating cells from the wound edge (starting point at $\mathrm{t}=0$ ) to the furthest migration point.

Multi-lineage differentiation of hPDLSCs. The cells were cultured in osteogenic, chondrogenic, adipogenic, and neurogenic differentiation media for 21 days (Gibco; Thermo Fisher Scientific, Inc.) to examine the osteogenic, chondrogenic, adipogenic, and neurogenic differentiation potential of hPDLSCs, respectively, when stimulated with an appropriate supplement. After 21 days of treatment, cells were stained with $2 \%$ alizarin red S stain (ARS) at $\mathrm{pH} 4.2,1 \%$ alcian blue, $0.3 \%$ oil red $\mathrm{O}$ dye (all three from Sigma-Aldrich; Merck KGaA), and Nissl stain. Stained cells were visualized under an inverted light microscope to detect the calcified matrix, proteoglycans, fat vacuoles, and Nissl bodies, which are indicators of osteogenic, chondrogenic, adipogenic, and neurogenic differentiation, respectively. 
Table I. Primer sequences for reverse transcription-quantitative polymerase chain reaction analysis.

\begin{tabular}{|c|c|c|}
\hline Gene & GenBank no. & Sequences \\
\hline Coll & NM007742 & $\begin{array}{l}\text { F: 5'-GCTCCTCTTAGGGGCCACT-3' } \\
\text { R: 5'-CCACGTCTCACCATTGGGG-3' }\end{array}$ \\
\hline Runx2 & NM_001146038 & $\begin{array}{l}\text { F: 5'-CGCACGACAACCGCACCAT-3' } \\
\text { R: 5'-CAGCACGGAGCACAGGAAGTT-3' }\end{array}$ \\
\hline$B S P$ & L09555 & $\begin{array}{l}\text { F: 5'-AACTTTTATGTCCCCCGTTGA-3' } \\
\text { R: 5'-TGGACTGGAAACCGTTTCAGA-3' }\end{array}$ \\
\hline$A L P$ & NM007431 & $\begin{array}{l}\text { F: 5'-CCAACTCTTTTGTGCCAGAGA-3' } \\
\text { R: 5'-GGCTACATTGGTGTTGAGCTTTT-3' }\end{array}$ \\
\hline$H P R T$ & NM_000194 & $\begin{array}{l}\text { F: 5'-GGCTATAAGTTCTTTGCTGACCTG-3' } \\
\text { R:5'-CCACAGGGACTAGAACACCTGCTA-3' }\end{array}$ \\
\hline
\end{tabular}

Coll, collagen 1; Runx2, runt-related transcription factor 2; BSP, bone sialoprotein; $A L P$, alkaline phosphatase; HPRT, hypoxanthine-guanine phosphoribosyl transferase; F, forward; R, reverse.

In vitro differentiation and mineralization. Cells $\left(4 \times 10^{4}\right)$ were cultured in a 24 -well plate with $\alpha$-MEM containing $10 \%$ FBS until they reached $50 \%$ to $60 \%$ confluency. The cells were fixed with $10 \%$ formalin solution (Duksan Chemical Co., Gyeonggi-do), followed by an incubation with $0.1 \%$ Triton X-100 for $5 \mathrm{~min}$. Then, the incubated cells were stained with a leukocyte ALP kit (Sigma-Aldrich; Merck $\mathrm{KGaA}$ ) according to the manufacturer's protocols. Mineralized nodules were detected by staining with $2 \%$ ARS at pH 4.2 on treatment day 14. For mineralization, cells were cultured in an osteogenic differentiation medium with $50 \mu \mathrm{g} / \mathrm{ml}$ ascorbic acid, $10 \mathrm{mM} \beta$-glycerophosphate, and $100 \mathrm{nM}$ dexamethasone (Sigma-Aldrich; Merck KGaA) for 14 days.

RNA isolation and reverse transcription-quantitative polymerase chain reaction $(R T-q P C R)$ analysis. We utilized RT-qPCR to evaluate the expression of osteogenesis-related genes in hPDLSCs $(22,23)$. Briefly, cells $\left(1 \times 10^{6}\right)$ were cultured in a 60 -mm culture dish for 2 weeks under differentiation induction conditions, and RNA was isolated from the treated cells using an RNeasy Mini kit (Qiagen) according to the manufacturer's instructions. Next, cDNA was synthesized from $2 \mu \mathrm{g}$ of total RNA using reverse transcriptase (Superscript II Preamplification System; Invitrogen; Thermo Fisher Scientific, Inc.). SYBR-Green PCR Master Mix (ABI Prism 7500; Applied Biosystems; Thermo Fisher Scientific, Inc.) was used for RT-qPCR. The experiment conditions were as follows: 40 cycles of denaturation for $15 \mathrm{sec}$ at $95^{\circ} \mathrm{C}$ and $1 \mathrm{~min}$ of amplification at $60^{\circ} \mathrm{C}$. All reactions were run in triplicate, and normalized to the housekeeping gene hypoxanthine-guanine phosphoribosyl transferase (HPRT). The cycle threshold values were calculated and compared to assess gene expression levels in control and gondre-treated groups. The relative mRNA expression levels in hPDLSCs and their gondre-treated counterparts were plotted in a histogram. We evaluated the expression levels of collagen 1 (Col1), runt-related transcription factor 2 (Runx2), bone sialoprotein (BSP), and alkaline phosphatase (ALP) and HPRT. The specific primer sets used for this analysis are listed in Table I.
Western blot analysis. Gondre-induced protein expression levels were determined with western blotting. For this, cells were lysed with RIPA buffer containing $1 \mathrm{mM}$ phenylmethylsulfonyl fluoride, centrifuged, and collected in clean Eppendorf tubes. Protein concentration was analyzed using the BSA protein assay kit (Bio-Rad Laboratories, Inc.). An equal volume of protein $(25 \mu \mathrm{g})$ was separated by sodium dodecyl sulfate-polyacrylamide gel electrophoresis and transferred to a polyvinylidene difluoride membrane (GE Healthcare). Primary antibodies against Runx2 and osterix (OSX) were purchased from Abcam. The blots were developed using horseradish peroxidase-conjugated secondary antibodies (Cell Signaling Technology, Inc.), and visualized using a gel-documentation imaging system (Chemi-Doc XRS+Imaging System; Bio-Rad Laboratories, Inc.). Blots were quantified using the ImageJ software (ImageJ v1.8, NIH), and normalized to $\alpha$-tubulin.

Statistical analysis. Statistical analysis was performed with one-way ANOVA using the Origin Pro 9.0 software. All experiments were performed in triplicate $(n=3)$, and the results are expressed as mean $\mathrm{OD} \pm \mathrm{SD}$. To compare significant differences between control and experimental groups, Tukey's post hoc analysis was performed. $\mathrm{P}<0.05$ was considered to indicate a statistically significant difference.

\section{Results and Discussion}

Characterization of the extract. The proton spectrum is a powerful analytical tool used to identify the different kinds of protons present in a structure. This information provides substantial support for explaining the chemical composition of a certain material. The ${ }^{1} \mathrm{H}-\mathrm{NMR}$ spectrum of the methanol-extracted sample is shown in Fig. 1. The NMR spectrum resembled the previously reported pattern of pectolinarin, which suggests that the extracted material primarily consisted of pectolinarin $(10,12)$. The proton NMR spectrum exhibits several peaks in the chemical shift (ठ) region of $0.83-2.2 \mathrm{ppm}$, which correspond to the methyl 


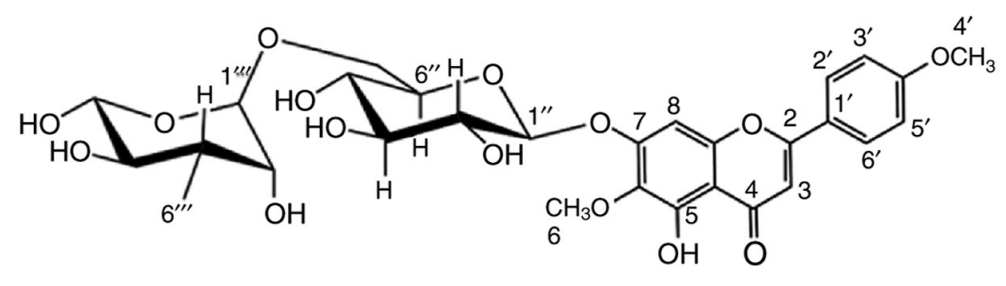

Pectolinarin
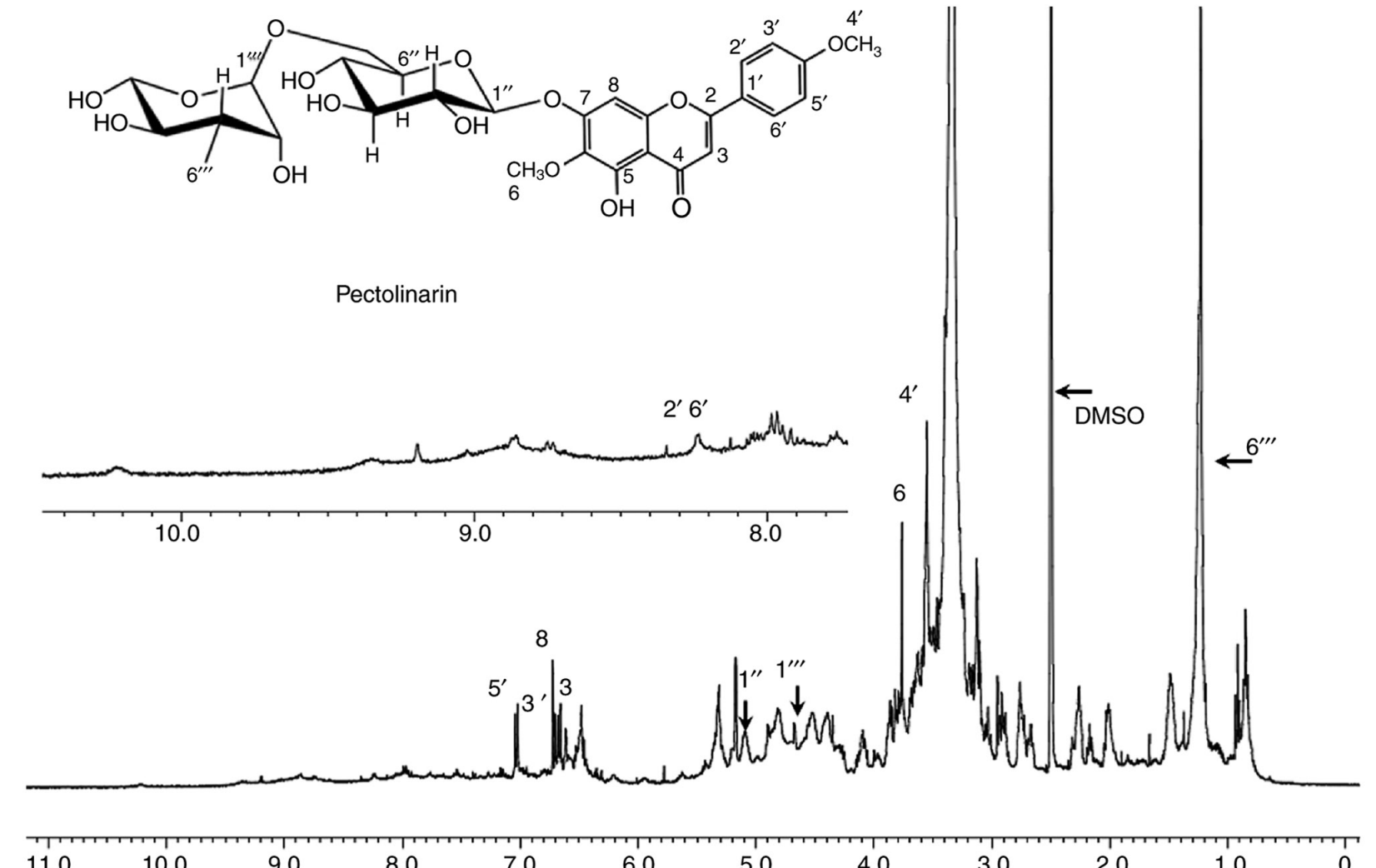

9.0

8.0

7.0

6.0

5.0

4.0

3.0

2.0

1.0

Figure 1. The ${ }^{1} \mathrm{H}$-nuclear magnetic resonance spectrum of the methanol-extracted bioactive phytochemicals from Cirsium setidens (gondre), indicating the presence of pectolinarin chemical moiety.

and methylene hydrogen moieties (24). Chemical shifts of other protons due to different chemical environments are indicated in detail in the proton spectrum. The MALDI-TOF mass spectrum of the methanol-extracted sample with a 2,5 -Dihydroxybenzoic acid (2,5-DHB)-assisted matrix is shown in Fig. 2. It is well established that the 2,5-DHB matrix enhances the intensity of the signals in the MALDI-TOF spectrometry (25). Interestingly, the gap between the two fragmented peaks is not constant. This is attributed to the presence of different chemical moieties in the extracted sample, which are associated with various linkages. The gap between the two peaks was $\sim 147$ and $149 \mathrm{Da}$, suggesting the presence of glucose and rhamnose units in the structure, respectively. It can also be inferred that it was linked with a glycosidic linkage, which was cleaved during the measurement. The appearance of a signal at $\sim 288$ shows the presence of a flavone structure in the methanol extract, which is linked to the glucose unit. It is not always necessary to consider the highest $\mathrm{m} / \mathrm{z}$ peak ratio as the molecular ion species $[\mathrm{M}+\mathrm{H}]^{+}$ in the positive mode, and $[\mathrm{M}-\mathrm{H}]^{-}$in a negative way, mainly because of the formation of molecular complexes $\left([2 \mathrm{M}+\mathrm{H}]^{+}\right.$ or $[2 \mathrm{M}-\mathrm{H}]^{-}$) or adducts with solvents or acid molecules (26). Based on the data obtained by ${ }^{1} \mathrm{H}-\mathrm{NMR}$ and MALDI-TOF, the methanol extract of gondre predominantly consisted of the pectolinarin chemical moiety.

Characterization of the hPDLSCs. A schematic illustration of the presence of periodontal ligament cells in the human molar tooth and of their isolation for primary cell culture is presented in Fig. 3A. Cultured cell morphology after different time intervals is shown in Fig. 3B. Stem cells are characterized by their pluripotentiality and their expression of different surface markers, and their fate is profoundly affected by growth factors, cytokines, and the surrounding microenvironments $(27,28)$. These factors can promote self-renewal and dedifferentiation of stem cells. This ability is known as the stemness potential (29). As shown in Fig. 3C, we evaluated the stemness potential of hPDLSCs through FACS analysis. Not only can hPDLSCs be differentiated into cementoblasts, osteoblasts, adipocytes, and chondrocytes (30), but they also exhibit superior bone cell formation properties, compared with human dental pulp stem cells and human periapical follicular stem cells (31). FACS results showed enhanced expression $(\sim 90 \%)$ of stem cell-related surface markers, such as CD13, CD90, and CD146, indicating the stemness potential of cultured hPDLSCs. The low expression $(\sim 10 \%)$ of CD34 surface markers in hPDLSCs supports their stemness potential as well. Fig. 3D shows the multi-lineage differentiation potential of hPDLSCs after 21 days of culture in diverse induction media, which was determined by monitoring the expression of specific markers. The presence of mineralized matrix, proteoglycan, fat vacuoles, and Nissl bodies, as detected by staining with ARS, alcian blue, oil red $\mathrm{O}$ dye, and Nissl stain, indicates the osteogenic, chondrogenic, adipogenic, and neurogenic potential of hPDLSCs, respectively (10).

Cell viability and migration. Cytotoxicity is one of the important criteria that determine whether a substance can be used in the biomedical field, since materials should be nontoxic 


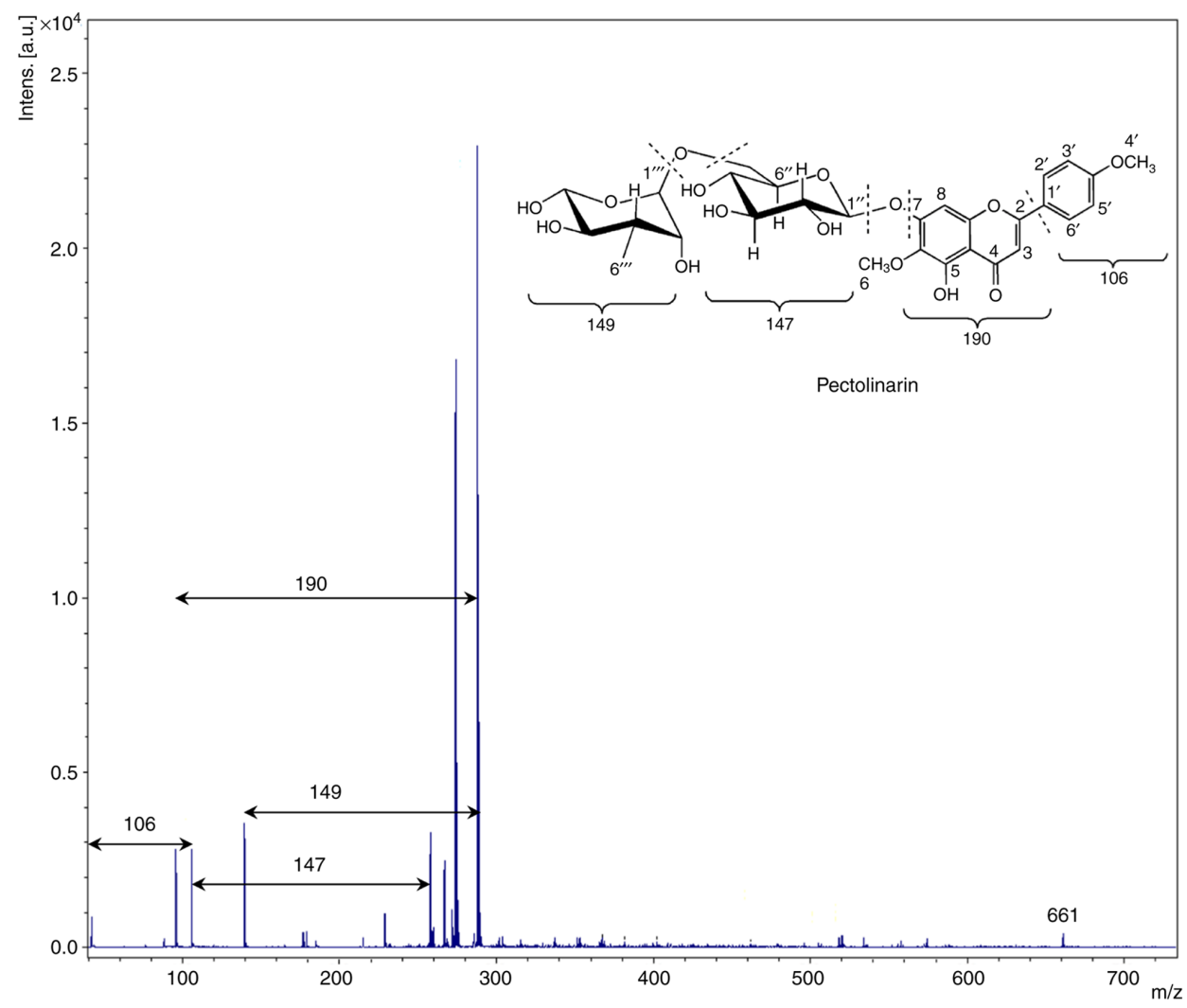

Figure 2. MALDI-TOF mass spectrum of the methanol-extracted sample of gondre, using 2,5-DHB as a matrix. The arrows indicate the gap between the two fragmented peaks in Da. The fragmented ions appeared at 98, 106, 139, 253, and 288 Da. MALDI-TOF, Matrix-assisted laser desorption/ionization (time-of-flight).
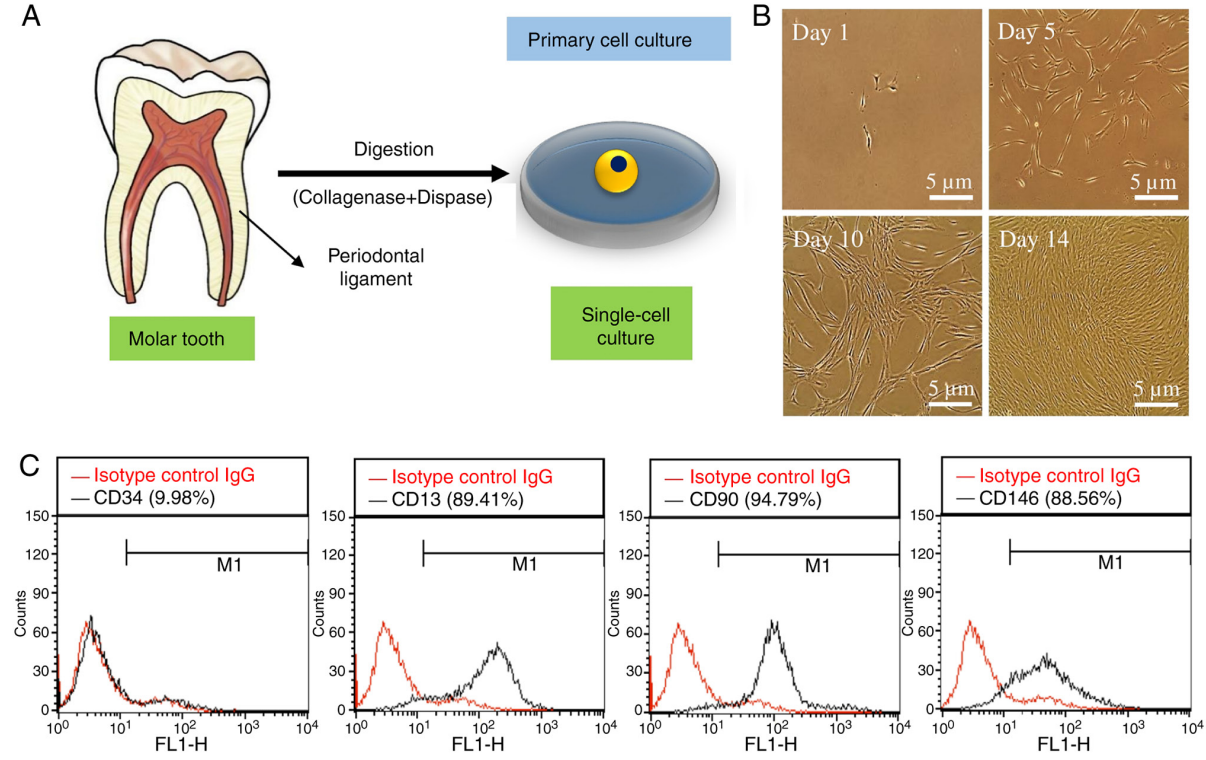

D

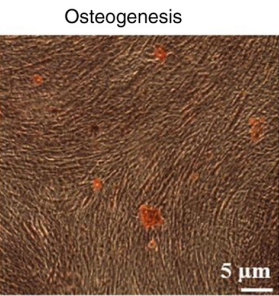

Chondrogenesis

Adipogenesis

Neurogenesis
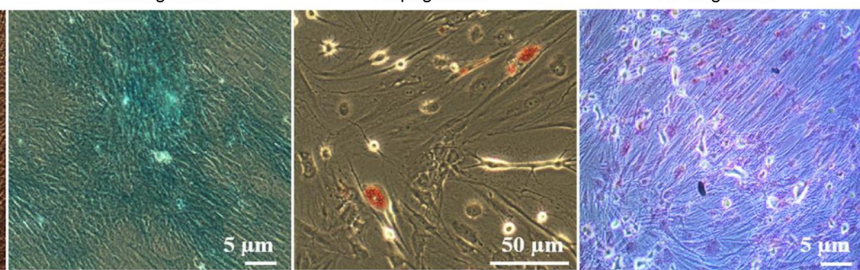

Figure 3. Isolation and characterization of hPDLSCs. (A) Schematic presentation for the presence of hPDLSCs in the human molar tooth, (B) The morphologies of the cultured stem cells after different time intervals, (C) Fluorescence-activated cell sorting analysis of hPDLSCs. (D) The multi-lineage differentiation potential of hPDLSCs after 21 days of treatment in different induction media. hPDLSCs, human periodontal ligament stem cells. 



Figure 4. Evaluation of the cytotoxicity and migration potential of hPDLSCs. (A) The cell viability data of hPDLSCs in the presence of different concentrations of gondre at indicated time intervals. (B and C) The scratch healing assay in the presence of gondre at indicated time intervals (magnification, $\mathrm{x} 10)$. The data are mean \pm SD of triplicate experiments $(\mathrm{n}=3)$. ${ }^{*} \mathrm{P}<0.05$ vs Control group. hPDLSCs, human periodontal ligament stem cells.

and biodegradable (32). The cytotoxic effects of gondre powder on hPDLSCs were evaluated using the MTT assay, as presented in Fig. 4A. Cells cultured in medium without gondre were used as control. An increase in cell viability in gondre-treated hPDLSCs, compared with control ones, was evidenced after $24 \mathrm{~h}$ of incubation, suggesting that there is biocompatibility. Furthermore, a significant improvement in cell viability was observed after $48 \mathrm{~h}$ of incubation, indicating their improved biocompatibility. This shows that gondre has no adverse effects on hPDLSCs. Among 0.01, 0.05, 0.1, 0.2, and $0.25 \%$ gondre concentrations, $0.05 \%$ elicited the highest cell viability, demonstrating that it is a suitable concentration for improved biocompatibility. Lee et al (15) also reported increased viability of hMSCs in the presence of gondre, after inducing oxidative stress with $\mathrm{H}_{2} \mathrm{O}_{2}$ for $8 \mathrm{~h}$. The authors noted that cell viability was significantly enhanced in the presence of $C$. setidens, compared to untreated conditions, which evidenced the protective effects of $C$. setidens against $\mathrm{H}_{2} \mathrm{O}_{2}$-induced oxidative stress (15).

Migration is essential for living cells, as it contributes to healthy development, and immune responses, as well as to disease processes, such as cancer metastasis and inflammation (33). Stem cell migration occurs not only during embryonic development but also in adult tissues to maintain homeostasis and repair damage. The migration ability of stem cells has tremendous therapeutic significance in the field of regenerative tissue applications (34). Fig. 4B and $\mathrm{C}$ shows the migration potential of hPDLSCs in the presence of different concentrations of gondre at indicated time intervals. Although cell migration towards the wounded area was initially slow in the presence of gondre compared with control conditions, after $24 \mathrm{~h}$, this tendency increased, and a higher accumulation of cells was observed in gondre-treated conditions, compared to the control. Moreover, a $0.05 \%$ concentration of gondre 
A
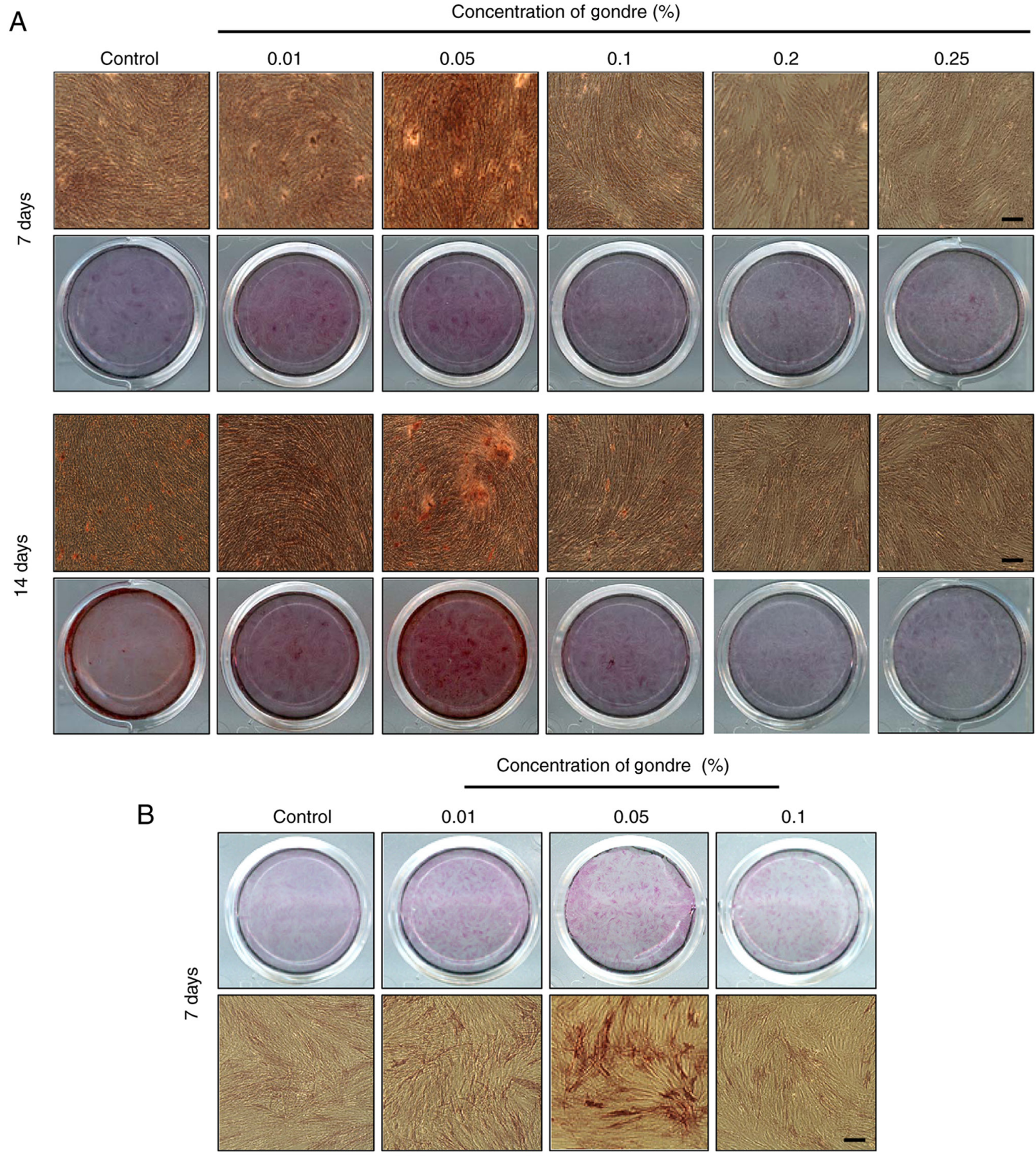

Figure 5. In vitro differentiation potential of hPDLSCs in the presence of gondre extract. (A) The mineralized nodule formation potential of hPDLSCs in the presence of different gondre concentrations at indicated time intervals (magnification, x10). (B) Alkaline phosphatase activity of hPDLSCs in the presence of the indicated gondre concentration after 14 days of incubation (magnification, x10). hPDLSCs, human periodontal ligament stem cells.

exhibited a high number of cells migrating towards the wound area. Cell viability and migration tendency results indicated that the $0.05 \%$ gondre concentration was the optimal concentration to improve cellular activity.

Mineralization and ALP activity. Stem cells are the most prominent cells in tissue engineering applications, owing to their differentiation potential. Even though they can be differentiated into osteoblasts, chondrocytes, adipocytes, and other cells $(35,36)$, their differentiation ability is profoundly affected by local biological conditions (37). Gondre-induced mineralization of hPDLSCs was examined through the ARS staining process after 7 and 14 days of incubation, and the results are shown in Fig. 5A. A more intense color was observed in gondre-treated cells compared with those in control conditions, which demonstrated better mineralization potential. This parameter is extensively affected by the biomaterial's concentration in the differentiation medium (38). Among experimental conditions $(0,0.01$, $0.05,0.1,0.2$, and $0.25 \%$ ), a gondre concentration of $0.05 \%$ showed greater potential for mineralization after 7 days of treatment. A similar trend was also observed after 14 days of 

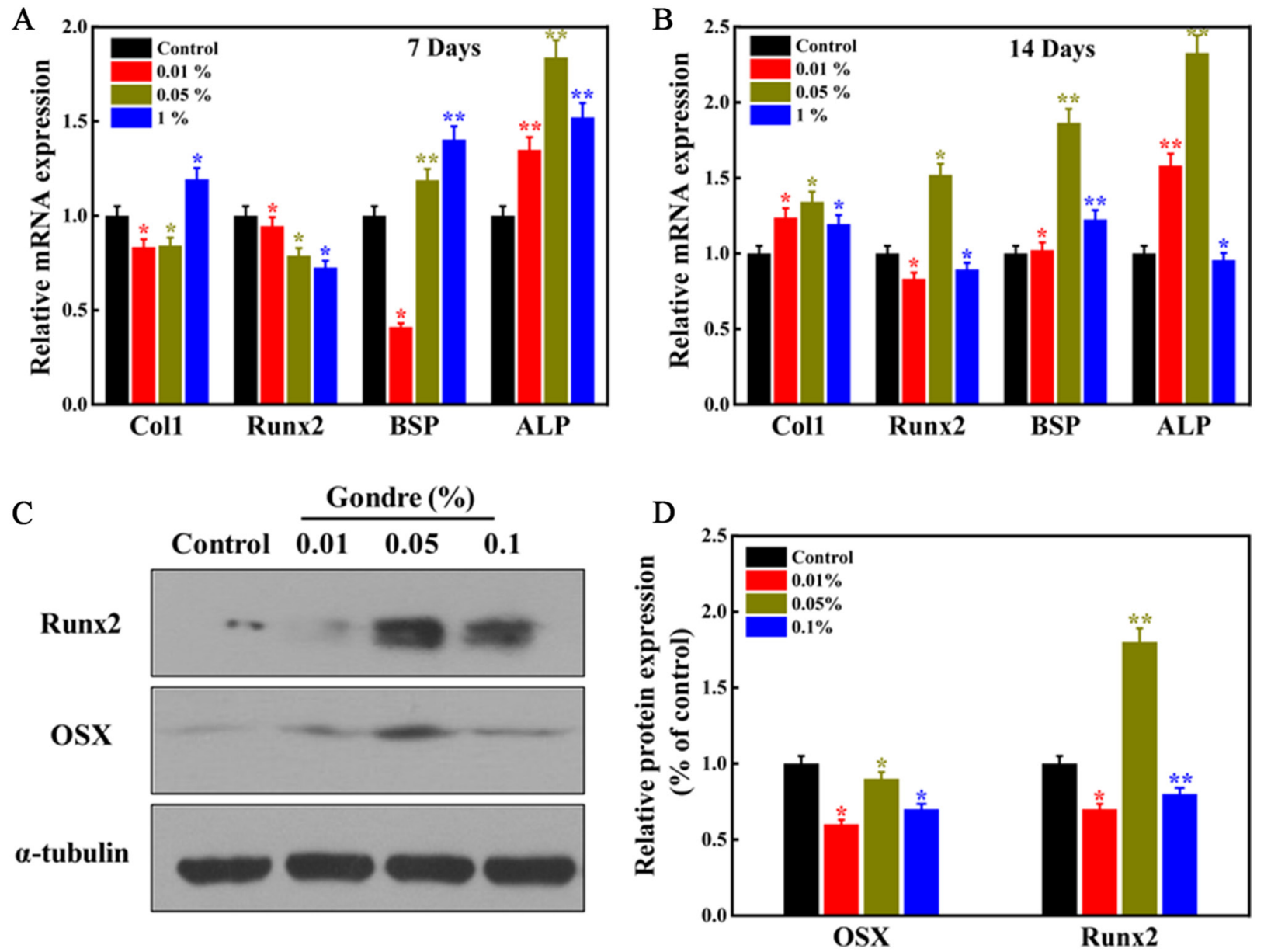

Figure 6. Evaluation of the expression of osteogenesis-specific gene markers and proteins in the presence of gondre powder. (A and B) The relative expression level of mRNA in the presence of different concentrations of gondre after 7, and after 14 days of treatment, respectively. Data were normalized to the housekeeping gene HPRT. (C) Western blot analysis of Runx2 and OSX following gondre (0, 0.01, 0.05, and 0.1\%) treatment. (D) Western blotting was quantified using the ImageJ software, and data were normalized to $\alpha$-tubulin. The data are mean $\pm \mathrm{SD}$ of triplicate experiments $(\mathrm{n}=3) .{ }^{*} \mathrm{P}<0.05,{ }^{* *} \mathrm{P}<0.01 \mathrm{vs}$. Control. Col1, collagen 1; Runx2, runt-related transcription factor 2; BSP, bone sialoprotein 2; ALP, alkaline phosphatase; OSX, osterix.

treatment, indicating that $0.05 \%$ is the optimal concentration for mineralization.

Different dosages of gondre may have different effects on stem cells. For instance, it has been reported that lower concentrations of this traditional herb enhance the metabolic efficacy and alkaline phosphatase activity, whereas high concentrations remarkably decrease cell density (3). Fig. 5B shows the ALP activity of hPDLSCs in the presence of gondre after 14 days of treatment. ALP activity was higher in the gondre-treated groups than in the control group, and this difference was significant in the $0.05 \%$ gondre-treated cells. Since ALP is an important gene marker that suggests the presence of preosteoblasts and osteoblasts for bone mineral production at the time of differentiation $(23,39)$, more intensely stained cells in the presence of gondre confirm a higher osteogenic potential of gondre-treated cells compared to control ones. It is well known that osteoclast resorption and osteoblast formation are two important processes that influence overall bone metabolic activity.

Osteogenic gene and protein expression. Bone formation is a complex biological process that involves several osteogenesis-associated genes (22). The expression of osteogenesis-related genes (Col1, Runx2, BSP, and ALP) in the presence of gondre after 7 and 14 days of treatment is shown in Fig. 6A and B. Untreated cells (no gondre) were considered as controls. Col1, the most abundant protein found in the bone matrix, is vital during the proliferation of osteoblast cells (40). The expression of the Coll biomarker from hPDLSCs in the presence of gondre evidences bone cell formation. Moreover, its expression was high in gondre-treated cells, compared to control ones, after 14 days of treatment, suggesting their greater osteogenic potential. Runx2 is another osteogenic marker that is expressed during osteogenesis. Since it is an early transcription marker, osteogenesis cannot occur without it. The results showed that its expression was higher in the gondre-treated cells than in the control ones. Moreover, Runx 2 expression was high after 14 days of treatment with $0.05 \%$ gondre. The expression and activity of Runx 2 are severely affected by other transcription factors and protein-protein or protein-DNA interactions. However, overexpression of the Runx 2 factor facilitates bone resorption (35). BSP comes from a 'small integrin-binding ligand $\mathrm{N}$-linked glycoprotein' (SIBLING) family, which occurs in bone and dentin. SIBLING plays a significant role in bone 
development, healing, remodeling, and mineralization (41). Higher expression levels of BSP in the presence of gondre, compared to those in control conditions, indicated a higher osteogenic efficiency.

Osteogenesis is widely affected by the nature, concentration, and size of the biomaterials present in the osteogenic media (42). Bi et al (43) tested the osteogenic potential of surface-functionalized gold nanoparticles (Au NPs) with different surface charges in the presence of hMSCs. They observed that Au NPs with hydroxyl groups exhibited higher osteogenic properties than others due to the presence of a hydroxyl moiety, which facilitates bone formation and growth, thus inducing osteogenesis. The hydroxyl groups also promote ECM growth, which contributes to osteogenesis (43). A similar result was also reported by Chen et al (44), with green tea catechins. In the present work, the abundant hydroxyl groups in pectolinarin facilitate apatite deposition in the matrix, as observed in the ALP activity and mineralization test, which causes greater osteogenesis. Cellular activity was higher at the $0.05 \%$ concentration compared to the others; hence, more apatite deposition occurred at this concentration, leading to superior osteogenesis.

Understanding the interaction mechanisms between hPDLSCs and gondre at the cellular and molecular level will help utilize the herb-derived material for osteogenic differentiation of cells in tissue engineering applications. Thus, the material characteristics and mechano-transduction pathways provide the primary molecular mechanics that affect cellular activities (45). It has been reported that nanomaterial-incorporated platforms display improved cellular activities, owing to their nano-topographical surface geometry, which promote cell proliferation and differentiation (46). Therefore, the abundance of hydroxyl groups in gondre promotes the improved osteogenic differentiation of hPDLSCs via their surface chemistry. As presented in Fig. 6C and D, changes in protein expression of hPDLSCs in the presence of gondre were evaluated with western blotting. Results showed that protein expression of Runx 2 was higher in gondre-treated cells than in control ones, further confirming their superior osteogenic potential. In particular, $0.05 \%$ gondre-treated cells presented the highest levels compared to the other groups. Runx2, also known as Cbfal or AML3 transcription factor, is an essential transcription factor that regulates the osteogenic differentiation of stem cells (47). Based on the improved osteogenic activity and protein expression in the presence of gondre, a hypothetical pathway is presented in Fig. 7. It has been reported that the mitogen-activated protein kinase (MAPK)/extracellular regulated protein kinase (ERK) signaling pathway may play a crucial role in osteogenic differentiation and bone cell development by accelerating Runx2 phosphorylation and transcriptional activity (47). Previously, it was reported that gondre extract had a positive role in $\mathrm{H}_{2} \mathrm{O}_{2}$-induced oxidative damage protection in hMSCs by signaling through MAPK/ERK pathway (15). Therefore, we assumed that the MAPK/ERK signaling pathway facilitates superior osteogenic differentiation in the presence of gondre, by activating Runx 2 phosphorylation and transcriptional activity. The lack of proper molecular mechanism to support

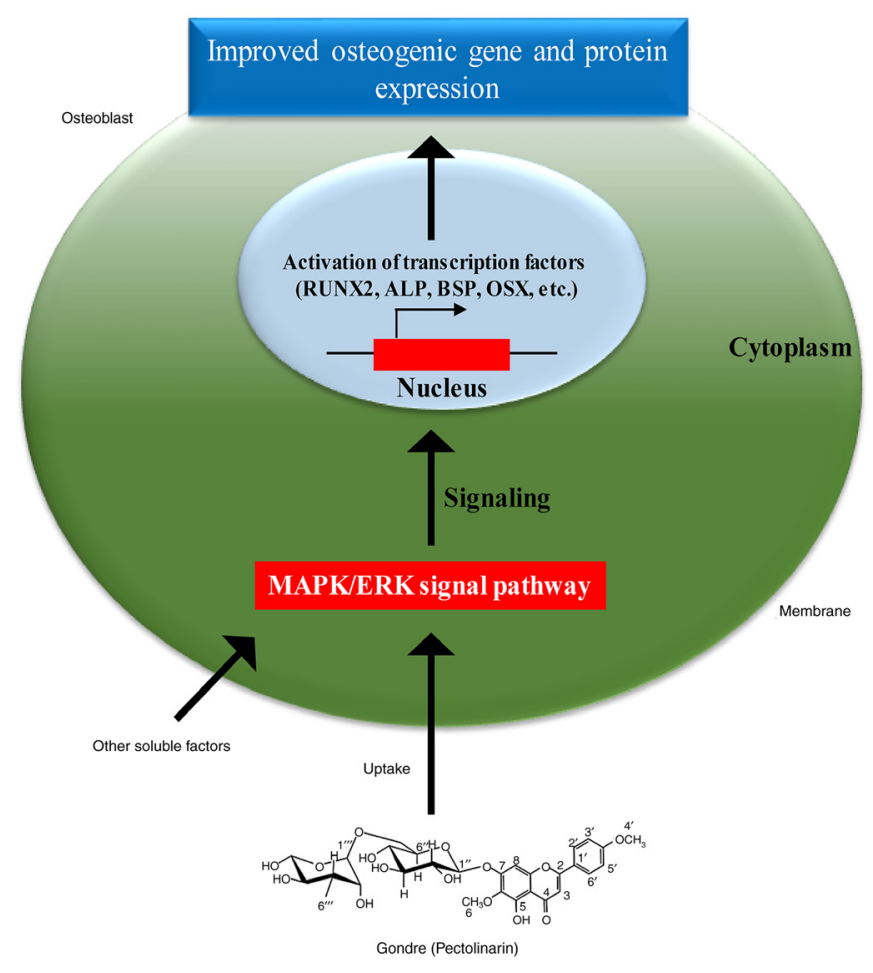

Figure 7. A hypothetical drawing for gondre-induced odontoblastic differentiation of hPDLSCs. hPDLSCs, human periodontal ligament stem cells; RUNX2, runt-related transcription factor 2; BSP, bone sialoprotein; ALP, alkaline phosphatase; OSX, osterix.

these findings will be the future direction of this work. In this study, a concentration of $0.05 \%$ gondre is optimal and can be explored as an osteogenic substrate for tissue engineering applications, especially in bone tissues.

In this study, we extracted the phytochemicals present in gondre, using methanol, and characterized them via MALDI-TOF mass spectrometry and ${ }^{1} \mathrm{H}-\mathrm{NMR}$ spectroscopy. It was observed that the methanol extract of gondre primarily consisted of pectolinarin. hPDLSCs were used to evaluate gondre cytotoxicity. The stemness potential of the hPDLSCs was evaluated using the FACS technique. Interestingly, the isolated hPDLSCs maintained their stemness potential. Notably, higher cell viability was observed in the presence of gondre, compared to the control, and viability was extensively affected by gondre content in the media. Among these concentrations $(0.01,0.05,0.1,0.2$, and $0.25 \%), 0.05 \%$ exhibited greater cell viability than the other concentrations. The cell migration study also supports that $0.05 \%$ is an ideal concentration for improved cellular activity. A more intense mineralized nodule formation occurred in the presence of $0.05 \%$ gondre concentration, and the control showed greater osteogenic potential. In addition, a significant enhancement in osteogenesis-related gene expression was observed in the presence of $0.05 \%$ concentration of gondre, compared to the other concentrations, after 7 and 14 days of treatment, suggesting its potential use in tissue engineering applications. Based on these findings, we concluded that gondre was effective for enhanced osteogenesis, and can be useful as a natural, edible, and osteogenic agent. 


\section{Acknowledgements}

The authors acknowledge the Central Instrumentation Laboratory, Kangwon National University, Chuncheon for providing the proton NMR and mass spectroscopy facility.

\section{Funding}

This research was supported by the Basic Science Research Program through the National Research Foundation of Korea (NRF) funded by the Ministry of Education (grant no. 2018R1A6A1A03025582) and the National Research Foundation of Korea (grant no. NRF-2019R1D1A3A03103828). The study was also supported by the Innovative Cultured Meat Technology Development Alchemist Project (grant no. 20012439) funded by the Ministry of Trade, Industry, and Energy (MoTIE, Korea).

\section{Availability of data and materials}

The datasets used and/or analyzed during the current study are available from the corresponding author on reasonable request.

\section{Authors' contributions}

SDD and DKP conceptualized the study, designed the methodology and validated the study. SDD, BJ, SIC and OHL performed formal analysis. SDD wrote the original draft. DKP reviewed and edited the manuscript. KTL conceptualized the work, acquired funding and gave the final approval of the paper. SDD and KTL confirm the authenticity of all the raw data. All authors read and approved the final manuscript.

\section{Ethics approval and consent to participate}

The present study was approved by the Institutional Review Board of the Dental Hospital, Seoul National University (Seoul, Republic of Korea; IRB Number 05004), and written consent was obtained from each patient.

\section{Patient consent for publication}

Not applicable.

\section{Competing interests}

The authors declare that they have no competing interests.

\section{References}

1. Ozen AE, Pons A and Tur JA: Worldwide consumption of functional foods: A systematic review. Nutr Rev 70: 472-481, 2012.

2. Biella CA, Salvador MJ, Dias DA, Dias-Baruffi M and Pereira-Crott LS: Evaluation of immunomodulatory and anti-inflammatory effects and phytochemical screening of Alternanthera tenella Colla (Amaranthaceae) aqueous extracts. Mem Inst Oswaldo Cruz 103: 569-577, 2008.

3. Cho BY, Park MR, Lee JH, Ra MJ, Han KC, Kang IJ and Lee OH: Standardized Cirsium setidens Nakai ethanolic extract suppresses adipogenesis and regulates lipid metabolisms in 3T3-L1 adipocytes and C57BL/6J mice fed high-fat diets. J Med Food 20: 763-776, 2017.
4. Daliri EBM, Choi SI, Cho BY, Jo HY, Kim SH, Chelliah R, Rubab M, Kim JH, Oh HT, Lee OH, et al: Biological activities of a garlic-Cirsium setidens Nakai blend fermented with Leuconostoc mesenteroides. Food Sci Nutr 7: 2024-2032, 2019.

5. Lee WB, Kwon HC, Cho OR, Lee KC, Choi SU, Baek NI and Lee KR: Phytochemical constituents of Cirsium setidens Nakai and their cytotoxicity against human cancer cell lines. Arch Pharm Res 25: 628-635, 2002.

6. Jung HA, Kim YS and Choi JS: Quantitative HPLC analysis of two key flavonoids and inhibitory activities against aldose reductase from different parts of the Korean thistle, Cirsium maackii. Food Chem Toxicol 47: 2790-2797, 2009.

7. Lee HB, Kwak JH, Zee OP and Yoo SJ: Flavonoids from Cirsium rhinoceros. Arch Pharm Res 17: 273-277, 1994.

8. Yim SH, Kim HJ and Lee IS: A polyacetylene and flavonoids from Cirsium rhinoceros. Arch Pharm Res 26: 128-131, 2003.

9. Park JC, Hur JM, Park JG, Kim SC, Park JR, Choi SH and Choi JW: Effects of methanol extract of Cirsium japonicum var. ussuriense and its principle, hispidulin-7-O-neohesperidoside on hepatic alcohol-metabolizing enzymes and lipid peroxidation in ethanol-treated rats. Phytother Res 18: 19-24, 2004.

10. Lim H, Son KH, Chang HW, Bae K, Kang SS and Kim HP: Anti-inflammatory activity of pectolinarigenin and pectolinarin isolated from Cirsium chanroenicum. Biol Pharm Bull 31: 2063-2067, 2008.

11. Jeong HC, Shim YS, Rhee YK, Choi SY, Hong HD, Chung J, Han MJ and Cho CW: Quantification of marker compounds in Cirsium setidens Nakai by HPLC-DAD. Food Sci Biotechnol 22: 1481-1486, 2013.

12. Yoo YM, Nam JH, Kim MY, Choi J and Park HJ: Pectolinarin and pectolinarigenin of Cirsium setidens prevent the hepatic injury in rats caused by D-galactosamine via an antioxidant mechanism. Biol Pharm Bull 31: 760-764, 2008.

13. Martínez-Vázquez M, Ramírez Apan TO, Lastra AL and Bye R: A comparative study of the analgesic and anti-inflammatory activities of pectolinarin isolated from Cirsium subcoriaceum and linarin isolated from Buddleia cordata. Planta Med 64: 134-137, 1998

14. Liu S, Luo X, Li D, Zhang J, Qiu D, Liu W, She L and Yang Z: Tumor inhibition and improved immunity in mice treated with flavone from Cirsium japonicum DC. Int Immunopharmacol 6: 1387-1393, 2006

15. Lee JH, Jung HK, Han YS, Yoon YM, Yun CW, Sun HY, Cho HW and Lee SH: Antioxidant effects of Cirsium setidens extract on oxidative stress in human mesenchymal stem cells. Mol Med Rep 14: 3777-3784, 2016.

16. Chamberlain G, Fox J, Ashton B and Middleton J: Concise review: Mesenchymal stem cells: Their phenotype, differentiation capacity, immunological features, and potential for homing. Stem Cells 25: 2739-2749, 2007.

17. Amiri F, Jahanian-Najafabadi A and Roudkenar MH: In vitro augmentation of mesenchymal stem cells viability in stressful microenvironments: In vitro augmentation of mesenchymal stem cells viability. Cell Stress Chaperones 20: 237-251, 2015.

18. Jo YY, Lee HJ, Kook SY, Choung HW, Park JY, Chung JH, Choung YH, Kim ES, Yang HC and Choung PH: Isolation and characterization of postnatal stem cells from human dental tissues. Tissue Eng 13: 767-773, 2007.

19. Seo BM, Miura M, Gronthos S, Bartold PM, Batouli S, Brahim J, Young M, Robey PG, Wang CY and Shi S: Investigation of multipotent postnatal stem cells from human periodontal ligament. Lancet 364: 149-155, 2004.

20. Jin B and Choung PH: Recombinant human plasminogen activator inhibitor-1 accelerates odontoblastic differentiation of human stem cells from apical papilla. Tissue Eng Part A 22: 721-732, 2016.

21. Huifang G, Jiang Y and Wang MH: Synergistic anti-diabetic effect of Cirsium setidens combined with other plants in vitro and in vivo. Korean J Plant Resour 28: 752-758, 2015.

22. Kim HB, Jin B, Patel DK, Kim JW, Kim J, Seonwoo H and Lim KT: Enhanced osteogenesis of human mesenchymal stem cells in presence of single-walled carbon nanotubes. IEEE Trans Nanobioscience 18: 463-468, 2019.

23. Dutta SD, Patel DK, Seo YR, Park CW, Lee SH, Kim JW, Kim J, Seonwoo H, and Lim KT: In vitro biocompatibility of electrospun poly ( $\varepsilon$-caprolactone)/cellulose nanocrystals-nanofibers for tissue engineering. J Nanomater 2019: 2061545, 2019.

24. Florencio-Silva R, Sasso GRdS, Sasso-Cerri E, Simões MJ and Cerri PS: Biology of bone tissue: Structure, function, and factors that influence bone cells. BioMed Res Int 2015: 421746, 2015. 
25. Seo YR, Patel DK, Shin WC, Sim WS, Lee OH and Lim KT: Structural elucidation and immune-enhancing effects of nove polysaccharide from Grifola frondosa. BioMed Res Int 2019: 7568609,2019

26. Cuyckens F and Claeys M: Mass spectrometry in the structural analysis of flavonoids. J Mass Spectrom 39: 1-15, 2004

27. Ouspenskaia T, Matos I, Mertz AF, Fiore VF and Fuchs E: WNT-SHH antagonism specifies and expands stem cells prior to niche formation. Cell 164: 156-169, 2016.

28. Guo G, von Meyenn F, Santos F, Chen Y, Reik W, Bertone P, Smith A and Nichols J: Naive pluripotent stem cells derived directly from isolated cells of the human inner cell mass. Stem Cell Reports 6: 437-446, 2016.

29. Aponte PM and Caicedo A: Stemness in cancer: Stem cells, cancer stem cells, and their microenvironment. Stem Cells Int 2017: 5619472, 2017.

30. Gay IC, Chen S and MacDougall M: Isolation and characterization of multipotent human periodontal ligament stem cells. Orthod Craniofac Res 10: 149-160, 2007.

31. Park JY, Jeon SH and Choung PH: Efficacy of periodontal stem cell transplantation in the treatment of advanced periodontitis. Cell Transplant 20: 271-285, 2011.

32. O'brien FJ: Biomaterials \& scaffolds for tissue engineering. Mater Today 14: 88-95, 2011.

33. Justus CR, Leffler N, Ruiz-Echevarria M and Yang LV: In vitro cell migration and invasion assays. J Vis Exp 88: e51046, 2014.

34. de Lucas B, Pérez LM and Gálvez BG: Importance and regulation of adult stem cell migration. J Cell Mol Med 22: 746-754, 2018.

35. Phinney DG: Functional heterogeneity of mesenchymal stem cells: Implications for cell therapy. J Cell Biochem 113 2806-2812, 2012.

36. Bianco P, Cao X, Frenette PS, Mao JJ, Robey PG, Simmons PJ and Wang CY: The meaning, the sense and the significance: Translating the science of mesenchymal stem cells into medicine. Nat Med 19: 35-42, 2013.

37. Giuliani N, Lisignoli G, Magnani M, Racano C, Bolzoni M, Palma BD, Spolzino A, Manferdini C, Abati C, Toscani D, et al: New insights into osteogenic and chondrogenic differentiation of human bone marrow mesenchymal stem cells and their potential clinical applications for bone regeneration in pediatric orthopaedics. Stem Cells Int 2013: 312501, 2013.

38. Simões LR, Maciel GM, Brandão GC, S Filho JD, Oliveira AB and Castilho RO: Chemical constituents of Distictella elongata (Vahl) Urb. (Bignoniaceae). An Acad Bras Cienc 85: 873-879, 2013.
39. Dutta SD, Hexiu J, Patel DK, Ganguly K and Lim KT: 3D-printed bioactive and biodegradable hydrogel scaffolds of alginate/gelatin/cellulose nanocrystals for tissue engineering. Int J Biol Macromol 167: 644-658, 2021

40. Niu C, Yuan K, Ma R, Gao L, Jiang W, Hu X, Lin W, Zhang X and Huang Z: Gold nanoparticles promote osteogenic differentiation of human periodontal ligament stem cells via the p38 MAPK signaling pathway. Mol Med Rep 16: 4879-4886, 2017.

41. Bouet G, Bouleftour W, Juignet L, Linossier MT, Thomas M, Vanden-Bossche A, Aubin JE, Vico L, Marchat D and Malaval L: The impairment of osteogenesis in bone sialoprotein (BSP) knockout calvaria cell cultures is cell density dependent. PLoS One 10: e0117402, 2015.

42. Li JJ, Kawazoe N and Chen G: Gold nanoparticles with different charge and moiety induce differential cell response on mesenchymal stem cell osteogenesis. Biomaterials 54: 226-236, 2015.

43. Bi X, You Z, Gao J, Fan X and Wang Y: A functional polyester carrying free hydroxyl groups promotes the mineralization of osteoblast and human mesenchymal stem cell extracellular matrix. Acta Biomater 10: 2814-2823, 2014.

44. Chen CH, Ho ML, Chang JK, Hung SH and Wang GJ: Green tea catechin enhances osteogenesis in a bone marrow mesenchymal stem cell line. Osteoporos Int 16: 2039-2045, 2005.

45. Zhao C, Tan A, Pastorin G and Ho HK: Nanomaterial scaffolds for stem cell proliferation and differentiation in tissue engineering. Biotechnol Adv 31: 654-668, 2013.

46. Wang G, Zheng L, Zhao H, Miao J, Sun C, Ren N, Wang J, Liu H and Tao X: In vitro assessment of the differentiation potential of bone marrow-derived mesenchymal stem cells on genipin-chitosan conjugation scaffold with surface hydroxyapatite nanostructure for bone tissue engineering. Tissue Eng Part A 17: 1341-1349, 2011.

47. Deng ZL, Sharff KA, Tang N, Song WX, Luo J, Luo X, Chen J, Bennett E, Reid R, Manning D, et al: Regulation of osteogenic differentiation during skeletal development. Front Biosci 13: 2001-2021, 2008.

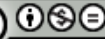

This work is licensed under a Creative Commons Attribution-NonCommercial-NoDerivatives 4.0 International (CC BY-NC-ND 4.0) License. 